\title{
Determination of FABP4, RBP4 and the MMP-9/NGAL complex in the serum of women with breast cancer
}

\author{
DIMITRIS TSAKOGIANNIS ${ }^{1}$, ELENI KALOGERA ${ }^{1}$, FLORA ZAGOURI ${ }^{2}$, \\ ELENI ZOGRAFOS $^{2}$, DIMITRIS BALALIS ${ }^{3}$ and GARYFALIA BLETSA ${ }^{1}$ \\ ${ }^{1}$ Research Center, Hellenic Anticancer Institute, Athens 10680; ${ }^{2}$ Department of Clinical Therapeutics, \\ Alexandra Hospital, National and Kapodistrian University of Athens School of Medicine, Athens 11528; \\ ${ }^{3}$ Department of Surgery, Saint Savvas, Anticancer Hospital, Athens 11522, Greece
}

Received April 6, 2020; Accepted July 31, 2020

DOI: $10.3892 / \mathrm{ol} .2020 .12346$

\begin{abstract}
Breast cancer is the most common type of cancer in females and is the leading cause of cancer-associated death among women, worldwide. The present study aimed to measure the serum levels of fatty acid-binding protein 4 (FABP4), retinol binding protein 4 (RBP4) and the MMP-9/neutrophil gelatinase-associated lipocalin (NGAL) complex in women diagnosed with breast cancer. Serum levels of the examined proteins were determined in the peripheral blood of patients via ELISA. Furthermore, whether the concentration of each protein was associated with breast cancer growth, molecular subtype, BMI, postmenopausal status, diabetes and the social background of patients was assessed. Women with invasive breast cancer demonstrated significantly higher levels of FABP4 $(\mathrm{P}=0.008)$. Additionally, considerably elevated FABP4 levels were demonstrated specifically in Luminal breast cancer cases $(\mathrm{P}<0.01)$. No significant association was recorded between RBP4 and breast cancer development. In addition, significantly lower levels of the MMP-9/NGAL complex were recorded in triple negative/HER-2 cases $(\mathrm{P}<0.05)$. BMI values appeared to influence the aforementioned associations, while significantly high serum levels of FABP4 and the MMP-9/NGAL complex were found in postmenopausal patients with breast cancer and a BMI $\geq 25 \mathrm{~kg} / \mathrm{m}^{2}(\mathrm{P}<0.05)$. In addition, high levels of FABP4 were significantly associated with breast cancer patients with diabetes $(\mathrm{P}=0.05)$. However, no association was identified between RBP4, the MMP-9/NGAL complex and diabetes. In conclusion, FABP4 can be regarded as a biomarker of breast cancer growth, while both FABP4 and the MMP-9/NGAL complex may provide considerable information regarding the development of specific breast cancer subtypes. FABP4 and
\end{abstract}

Correspondence to: Dr Garyfalia Bletsa, Research Center, Hellenic Anticancer Institute, Valtetsiou 11, Athens 10680, Greece E-mail: rdc@antikarkiniko.gr

Key words: breast cancer, fatty acid-binding protein 4, retinol binding protein 4, MMP-9/neutrophil gelatinase-associated lipocalin, molecular subtype the MMP-9/NGAL complex may also be able to predict the development of breast cancer in postmenopausal patients with obesity.

\section{Introduction}

Breast cancer is the most frequent developing malignancy among women with high mortality rates, worldwide $(1,2)$. Epidemiological data revealed that in 2018 more than 2 million women were diagnosed with breast cancer, accounting for more than 600.000 cancer-associated deaths (2-4). Since 1980, the global prevalence of breast cancer provides a consistently positive trend and it has been anticipated that the tendency for the number of incidences to rise will continue in the following years (2-4). Although the $60 \%$ of deaths occur in developing countries, the breast cancer mortality rate has been reduced in most developed countries due to the establishment of novel methodologies in screening, diagnosis and cancer therapy $(5,6)$.

Over the last years, several studies have focused on the analysis of numerous biomarkers, in order to improve the early prognosis, diagnosis and the proper breast cancer treatment (7-9). In particular, the effect of fatty acid-binding protein 4 (FABP4) on breast disease gained increased popularity, since high levels of the corresponding protein have been observed in plasma of breast cancer patients, while exogenous FABP4 significantly stimulates the proliferation of breast cancer cells (10-12). FABP4 is a member of fatty acid-binding proteins family (FABPs) which is implicated in the intracellular transportation of fatty acids in several cellular organelles (12). FABP4 is constantly expressed in macrophage and adipocyte, while it is involved in numerous cellular functions, including fatty acid uptake and storage as well as in regulation of gene expression, cell proliferation and differentiation $(12,13)$. In macrophages FABP4 regulates inflammatory responses via activation of the NF- $\kappa \mathrm{B}$ (nuclear factor $\kappa \mathrm{B}$ ) and JNK pathways (c-Jun N-terminal kinase), while in adipocytes FABP4 stimulates lipolysis and constraints lipogenesis through interaction with HSL (hormone-sensitive lipase) and PPAR $\gamma$ (peroxisome proliferator-activated receptor gamma) (14-16). In regard to breast cancer development FABP4 is highly expressed in a small subgroup of tumor associated macrophages (TAMs). FABP4-positive TAM subgroup in turn assembles in the late 
stage of mammary tumor, stimulating tumor growth, (17). An alternative pathway implicates adipocytes in cases of obesity. In particular, during obesity adipocytes release FABP4 which triggers oncogenic signaling factors, including IL-6, STAT3 and ALDH1 in breast mammary tumor cells, leading to tumor progression (14,16-18).

Recent analyses have focused on the implication of retinol binding protein 4 (RBP4) in breast cancer development, as well. RBP4 is an adipokine and member of the lipocalin family of proteins $(19,20)$. Lipocalin protein family consist of a heterogeneous group of extracellular proteins, expressed in liver and adipose tissue and they facilitate the transferring of small hydrophobic molecules, including hormones, prostaglandins, arachidonic acids and retinoids, while it is the major transporter for retinol acid (Vitamin A) $(20,21)$. RBP4 along with retinol acid (Vitamin A) stimulate STRA6 that in turn recruit and activate Janus kinase and the transcriptional factors STAT3 or STAT5 (22). Alternatively, RBP4 alone can activate pro-inflammatory responses through JNK1, JNK2 or Toll-like receptors (23). The expression of RBP4 is associated with insulin resistance as well as with cardiovascular risk markers, including body mass index [BMI; calculated as weight $(\mathrm{kg})$ divided by height $\left(\mathrm{m}^{2}\right)$ ], waist to hip ratio and the levels of triglyceride in serum (21). Furthermore, RBP4 is correlated with numerous types of cancer, such as prostate cancer, colon adenoma, ovarian cancer and oral cancer (24-26), but its association with breast cancer growth remains rather vague $(27,28)$.

Finally, neutrophil gelatinase-associated lipocalin (NGAL) is a member of the lipocalin family and it has been related with breast disease, as well $(20,29)$. NGAL is a fundamental component of cytoplasmic granules of human neutrophils and it contributes to the chelation of bacterial siderophores, thus inhibiting iron availability and consequently it prevents bacteria to establish infection $(30,31)$. Moreover, NGAL act as siderocalin and with sideropores and ferrous iron $\left(\mathrm{Fe}^{++}\right)$contribute to iron uptake that is necessary in regulating the iron-dependent growth pathways (32). Cancer cells have increased need for intracellular iron, while iron uptake is pivotal in regulating cell proliferation, invasion and metastasis $(32,33)$. NGAL expression is regarded as a considerable diagnostic and prognostic biomarker in several diseases, including inflammation and tumor growth $(29,31,34)$. It is noteworthy that NGAL generates a complex with metalloproteinase-9 (MMP-9) that constraints MMP-9 autodegradation and subsequently leads to increased activity of MMP-9 (31,34-36). Previous studies have suggested that MMP-9/NGAL complex augments cancer growth, including breast cancer (29,31,34-36). MMP-9 is found in gelatinase granules and contribute to cancer progression, invasion and metastasis in numerous neoplastic diseases $(29,37,38)$. Notably, a previous analysis in Greek population demonstrated that high levels of MMP-9, NGAL as well as increased levels of MMP-9/NGAL complex are associated with breast disease (26).

Nowadays, no further analyses have been conducted in order to better evaluate the influence of MMP-9/NGAL complex on the development of breast malignancy, while little is known concerning the impact of FABP4 and RBP4 on patients' susceptibility to the development of breast cancer in Greek women. Towards this end, the present study focused on the relationship of serum levels of FABP4, RBP4,
MMP-9/NGAL complex with the growth of breast cancer in the Greek population. Moreover, we examined the association of FABP4, RBP4, MMP-9/NGAL complex with the different breast cancer molecular subtypes, body mass index (BMI), diabetes, menopausal status and the social background of patients. Our goal was to elucidate whether the examined proteins are implicated in breast cancer development as well as to further investigate whether additional factors may influence this association, providing valuable information concerning the prognosis and diagnosis of breast cancer.

\section{Materials and methods}

Breast samples. In the present analysis a total of 73 women were examined. In particular, 53 women were diagnosed with breast cancer and they were further classified into different molecular types according to their immunehistochemistry (IHC) profile concerning ER (estrogen receptor), PR (progesterone receptor), HER-2 (human epidermal growth factor receptor 2) and ki67 $(39,40)$. As a result, patients were characterized as Luminal A $(n=22)$, Luminal B $(n=8)$, Triple Negative-TN: ER negative, PR negative, HER-2 negative $(n=15)$ and HER-2 positive: ER negative, PR negative, HER-2 positive $(n=8)$. According to histopathological criteria all breast cancer cases were characterized as invasive ductal carcinoma (IDC). Moreover, the control group comprised 20 healthy women. The mean age of the examined patients and controls was $62.8 \pm 14.12$ and $58.7 \pm 7.9$ years, respectively, while the mean age in Luminal $64 \pm 12$ years old and the mean age in TN/HER-2 group was $61 \pm 14.12$ years old. In addition, 44 patients were postmenopausal and 9 were premenopausal, while 45 breast cancer patients were diagnosed with diabetes, as well. Finally, the mean BMI value of breast cancer cases was $28.1 \pm 6 \mathrm{~kg} / \mathrm{m}^{2}$, while the mean BMI value of healthy women was $27.7 \pm 4.2 \mathrm{~kg} / \mathrm{m}^{2}$. Clinical samples were collected from Anticancer Oncology Hospital of Athens 'Saint Savvas' between May 2017 and July 2018. All patients signed an informed consent form, while the study was approved from the Research Committee of the Hospital.

Sample preparation. Venus blood was collected from patients and controls between 12:00 a.m. and 14:00 p.m. Sampling was performed directly into serum vacuum tubes with clot activator, while prior to centrifugation, tubes remained at room temperature for 20-30 min to enable blood clotting. Subsequently, the samples were centrifuged at 3,000 x g for $15 \mathrm{~min}$ at $8^{\circ} \mathrm{C}$, the serum was isolated and divided into aliquots parts, while all serums were stored at $-80^{\circ} \mathrm{C}$ for further use.

ELISA assay for the measurement of serum levels of FABP4, RBP4 and MMP-9/NGAL complex. The evaluation of serum levels of FABP4, RBP4 and MMP-9/NGAL complex in the examined clinical samples was conducted for each individual protein in duplicates through enzyme-linked immunosorbent assay (ELISA), using the respective Human FABP4, RBP4 and MMP-9/NGAL Quantikine ${ }^{\circledR}$ ELISA kit (R\&D Systems), according to the manufacturers instruction. Finally, the acquired fluorescence data were analyzed using Multiscan ${ }^{\mathrm{TM}}$ FC Microplate Photometer (Thermo Fisher Scientific, Inc.). 
Statistical analysis. Data are presented with mean, standard deviation and median, while statistical analysis was conducted considering whether they followed a normal distribution or not, respectively. Normality was examined through D'Agostino - Pearson, Shapiro-Wilk or Kolmogorov-Smirnov tests. In particular, FABP4 data follow a non normal distribution, whereas RBP4 and MMP-9/NGAL complex data follow a normal distribution. Student's t-test or Mann-Whitney non-parametric test were used to examine the association of the respective proteins with breast cancer development, BMI, menopausal status, diabetes and social background of patients. One way Anova was used for statistical analysis of the expression of RBP4, MMP-9/NGAL in Luminal, TN/HER-2 cancers and controls, followed by the Tukey post hoc test for pair-wise comparison, while Kruskal-Wallis non parametric test was performed for statistical analysis of the expression of FABP4 in the respective breast cancer molecular subtypes, followed by the Dunn multiple comparison post hoc test. $\mathrm{P}$ values were regarded as statistically significant at the 0.05 cut off level. All analyses were carried out with the GraphPad Prism 6 (GraphPad Software, Inc.).

\section{Results}

FABP4, RBP4, MMP9/NGAL complex and breast cancer. In the present analysis the serum levels of FABP4, RBP4 and MMP9/NGAL complex were measured in a total of 73 women, 53 of which have been diagnosed with breast cancer, while 20 cases were used as control group. The differences that occur at protein levels between patients and controls were evaluated, in order to investigate whether the concentrations of FABP4, RBP4 and MMP9/NGAL complex are related with breast cancer in the studied population. The mean and median serum concentrations of the respective proteins are presented in Table I. According to our results it was demonstrated that the median concentration of FABP4 in breast cancer cases was significantly higher than that of healthy women (median concentration; $27,740 \mathrm{pg} / \mathrm{ml}$ vs. 22,115 pg/ml, P=0.0078) (Tables I and II, Fig. 1). However, no considerable association was recorded between cancer cases and controls, regarding the serum levels of RBP4 and MMP9/NGAL complex, respectively (Tables I and II; Fig. 1).

FABP4, RBP4, MMP9/NGAL complex and molecular subtypes. The relationship of the corresponding proteins with the development of breast disease was further examined considering the molecular classification of breast cancer into different molecular subtypes $(34,35)$. Hence, the examined cases were classified into two major groups, containing the Luminal cohort (Luminal A and Luminal B) and TN/HER-2 group (HER-2 positive and Triple Negative). In regard to FABP4 serum levels, it was demonstrated that the median concentration of the respective protein in Luminal cases was considerably higher than that of healthy women (median concentration; $37,105 \mathrm{pg} / \mathrm{ml}$ vs. $22,115 \mathrm{pg} / \mathrm{ml}, \mathrm{P}=0.0002$ ), while high levels of FABP4 were recorded in Luminal group when compared with that of TN/HER-2 group (median concentration; 37,105 pg/ml vs. $25,040 \mathrm{pg} / \mathrm{ml}, \mathrm{P}=0.0073$ ) (Tables I and II; Fig. 2). Although the median concentration of FABP4 in TN/HER-2 cluster was found to be increased compared to healthy women, the difference was not considered as statistically significant $(\mathrm{P}=\mathrm{ns})$. In addition, no significant association was recorded between the groups of breast cancer cases and the serum levels of RBP4 protein (Tables I and II, Fig. 2).

Interestingly, differences in serum levels of MMP9/NGAL complex were found between breast cancer subtypes. In particular, slightly elevated levels of MMP9/NGAL complex were recorded in Luminal cohort compared to control group, but this association did not reach the limits of statistical significance (mean concentration-ng/ml; 50.97 \pm 4.191 vs. 47.96 $\pm 7.566, \mathrm{P}=\mathrm{ns}$ ) (Tables I and II). In contrast, the serum levels of MMP-9/NGAL complex were found to be substantially decreased in TN/HER-2 group. In particular, the mean concentration of MMP-9/NGAL complex in TN/HER-2 group was considerably lower than that of control group (mean concentration-ng/ml; $28.59 \pm 3.78$ vs. $47.96 \pm 7.566, \mathrm{P}=0.03$ ) and Luminal cohort, as well (mean concentration-ng/ml; $28.59 \pm 3.78$ vs. 50.97 $\pm 4.191, \mathrm{P}=0.0002$ ) (Tables I and II; Fig. 2).

FABP4, RBP4, MMP9/NGAL complex and BMI. A stratified analysis according to body mass index (BMI) values was performed in order to evaluate whether the BMI in combination with serum levels of FABP4, RBP4 and MMP9/NGAL complex, may be used as valuable biomarkers for the development of breast disease. In particular, breast cancer cases and controls were classified into two groups, comprising specimens with BMI values $\geq 25 \mathrm{~kg} / \mathrm{m}^{2}$ and specimens with BMI values $<25 \mathrm{~kg} / \mathrm{m}^{2}$ (Tables III and IV). Our results indicate that in clinical samples with $\mathrm{BMI} \geq 25 \mathrm{~kg} / \mathrm{m}^{2}$ the median concentration of FABP4 was significantly higher when compared with that of healthy women (median concentration $37,180 \mathrm{pg} / \mathrm{ml} \mathrm{vs}$. 24,445 pg/ml, $\mathrm{P}=0.0023$ ) (Tables III and IV). In contrast, in samples with BMI $<25 \mathrm{~kg} / \mathrm{m}^{2}$ no significant alterations were detected in FABP4 levels between cancer and control cases. Moreover, no significant differences were recorded in RBP4 and MMP9/NGAL serum levels between cancer cases and controls, neither when $\mathrm{BMI} \geq 25 \mathrm{~kg} / \mathrm{m}^{2}$ nor when $\mathrm{BMI}<25 \mathrm{~kg} / \mathrm{m}^{2}$. Merging outcomes derived from BMI clusters and the different cancer groups it was revealed that when BMI $\geq 25 \mathrm{~kg} / \mathrm{m}^{2}$ the median concentration of FABP4 was significantly augmented in Luminal cases compared either to TN/HER-2 (median concentration $45,595 \mathrm{pg} / \mathrm{ml}$ vs. $22,960 \mathrm{pg} / \mathrm{ml}, \mathrm{P}=0.018$ ) or healthy women (median concentration $45,595 \mathrm{pg} / \mathrm{ml}$ vs. $24,445 \mathrm{pg} / \mathrm{ml}, \mathrm{P}=0.0009$ ) (Tables III and IV). However, no considerable changes in FABP4 levels were detected in cases with BMI lower than $25 \mathrm{~kg} / \mathrm{m}^{2}$. In regard to RBP4 serum levels, no significant associations were identified between the concentration of the respective protein and BMI groups within the different molecular subtypes of breast disease (Tables III and IV). Finally, considering results derived from MMP9/NGAL complex, it was observed that in TN/HER-2 cases with $\mathrm{BMI} \geq 25 \mathrm{~kg} / \mathrm{m}^{2}$ the mean concentration of the respective complex was significantly lower than that of Luminal samples (mean concentration-ng/ml; 30.59 \pm 6.45 vs. $50.83 \pm 3.7, \mathrm{P}=0.014$ ) (Tables III and IV). Nevertheless, no further associations were found between the concentration of MMP9/NGAL complex and BMI cohorts within the divergent subtypes of breast malignancy (Tables III and IV).

FABP4, RBP4, MMP9/NGAL complex, menopausal status, diabetes and social background. Finally, we further evaluate 


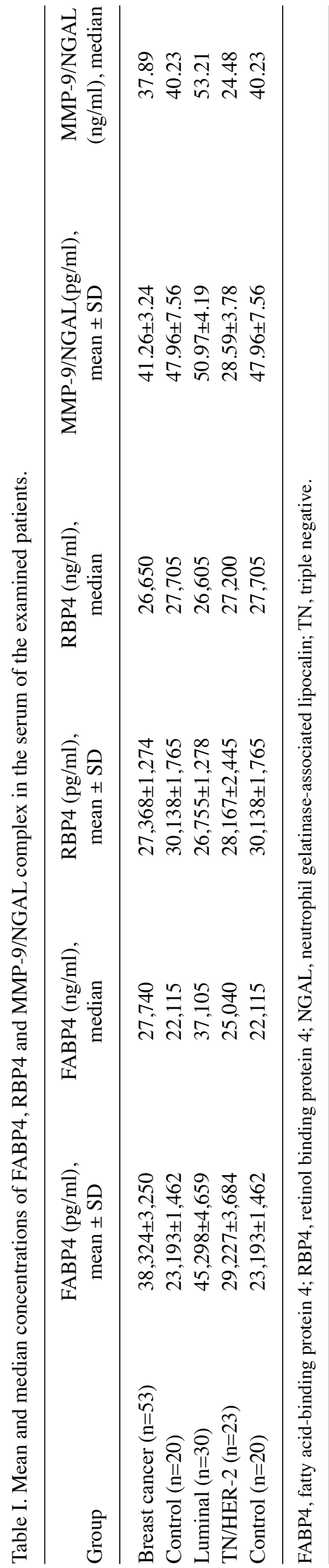

Table II. P-values were calculated to estimate the differences in protein levels between patients with breast cancer and controls, and among different breast cancer subtypes.

\begin{tabular}{lrrr}
\hline & \multicolumn{3}{c}{ P-values } \\
\cline { 2 - 4 } Groups & FABP4 & RBP4 & MMP-9/ \\
& 0.008 & 0.260 & 0.420 \\
\hline Breast cancer vs. control & $<0.001$ & 0.130 & 0.700 \\
Luminal vs. control & 0.420 & 0.320 & 0.030 \\
TN/HER-2 vs. control & 0.007 & 0.600 & $<0.001$ \\
Luminal vs. TN/HER-2 & & & \\
\hline
\end{tabular}

FABP4, fatty acid-binding protein 4 ; RBP4, retinol binding protein 4 ; NGAL, neutrophil gelatinase-associated lipocalin; TN, triple negative.

the relationship of the tested serum proteins with menopausal status, diabetes, and social background of the examined patients including smoking and exercise. Considering our results, it was observed that serum FABP4 levels are significantly increased in postmenopausal breast cancer patients when compared with that of premenopausal patients (median concentration $32,720 \mathrm{pg} / \mathrm{ml}$ vs. $14,550 \mathrm{pg} / \mathrm{ml}, \mathrm{P}=0.0005$ ) (Table V). In addition, in the cohort of postmenopausal patients the concentration of FABP4 protein is significantly higher in individuals with $\mathrm{BMI} \geq 25 \mathrm{~kg} / \mathrm{m}^{2}$ than patients with $\mathrm{BMI}<25 \mathrm{~kg} / \mathrm{m}^{2}$ (median concentration $40,050 \mathrm{pg} / \mathrm{ml}$ vs. $24,610 \mathrm{pg} / \mathrm{ml}, \mathrm{P}=0.03$ ) (Table VI). In addition, the link of FABP4 with diabetes reached the limits of statistical significance, as the serum FABP4 concentration was found to be higher in breast cancer patients with diabetes when compared with that of patients with no diabetes (median concentration $54,310 \mathrm{pg} / \mathrm{ml}$ vs. $27,460 \mathrm{pg} / \mathrm{ml}, \mathrm{P}=0.05$ ) (Table VII).

Although the serum levels of MMP9/NGAL complex are not modified considering the menopausal status of patients, it was observed that MMP9/NGAL complex in postmenopausal individuals is significantly higher in patients with BMI $\geq 25 \mathrm{~kg} / \mathrm{m}^{2}$ than patients with BMI $<25 \mathrm{~kg} / \mathrm{m}^{2}$ (mean concentration $\mathrm{ng} / \mathrm{ml}$; $46.21 \pm 3.51$ vs. $30.34 \pm 5.15, \mathrm{P}=0.016$ ) (Table VI). Moreover, no significant association was recorded between MMP9/NGAL complex and diabetes in breast cancer patients as well as no significant association was detected regarding RBP4 serum levels either with menopausal status or diabetes (Table VII). Furthermore, it was revealed that smoking and exercise do not influence the serum levels of FABP4, RBP4 and MMP9/NGAL in breast cancer patients.

\section{Discussion}

The stratification of breast cancer severity lies on numerous factors, including TNM stage, tumor grade, lymphatic invasion as well as the expression of the hormonal receptors ER, PR and HER-2 $(39,40)$. Although these factors offer crucial information concerning the risk profile of patients and they significantly contribute to select the most suitable therapeutic approach $(41,42)$, today, there is a growing interest in 


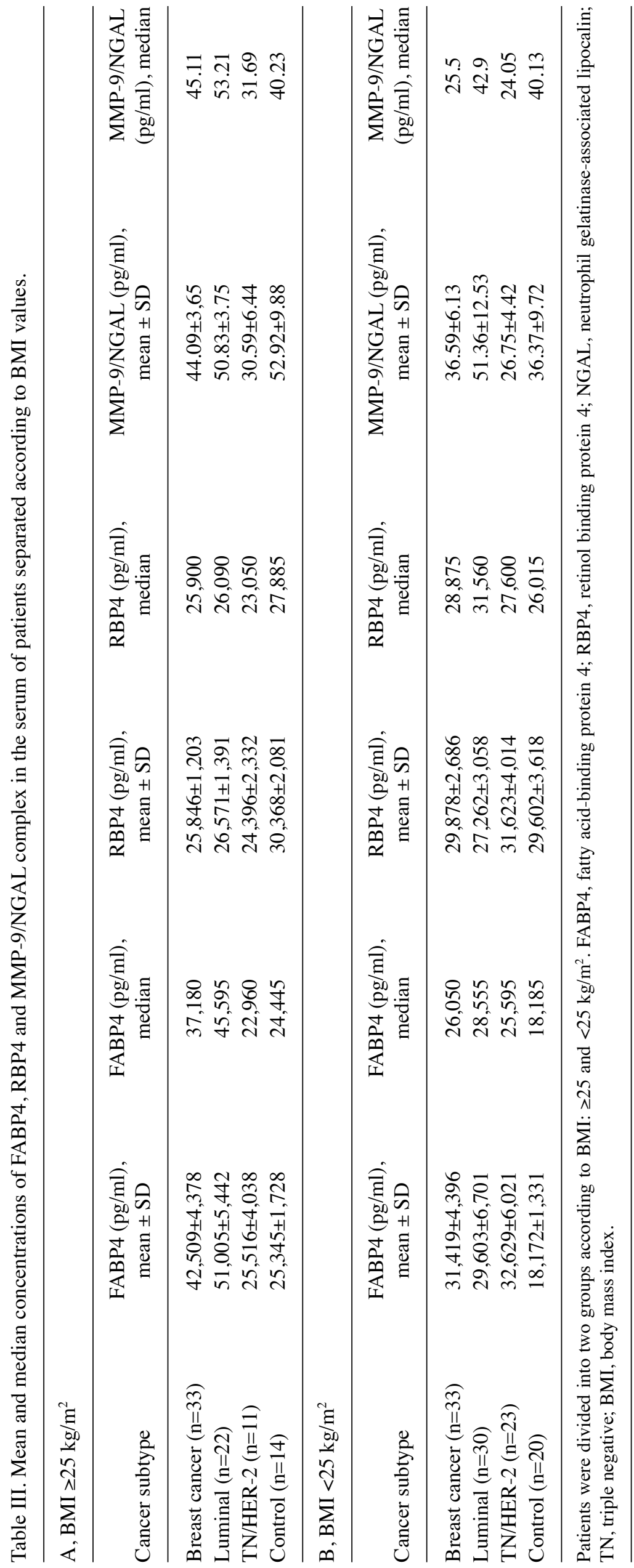



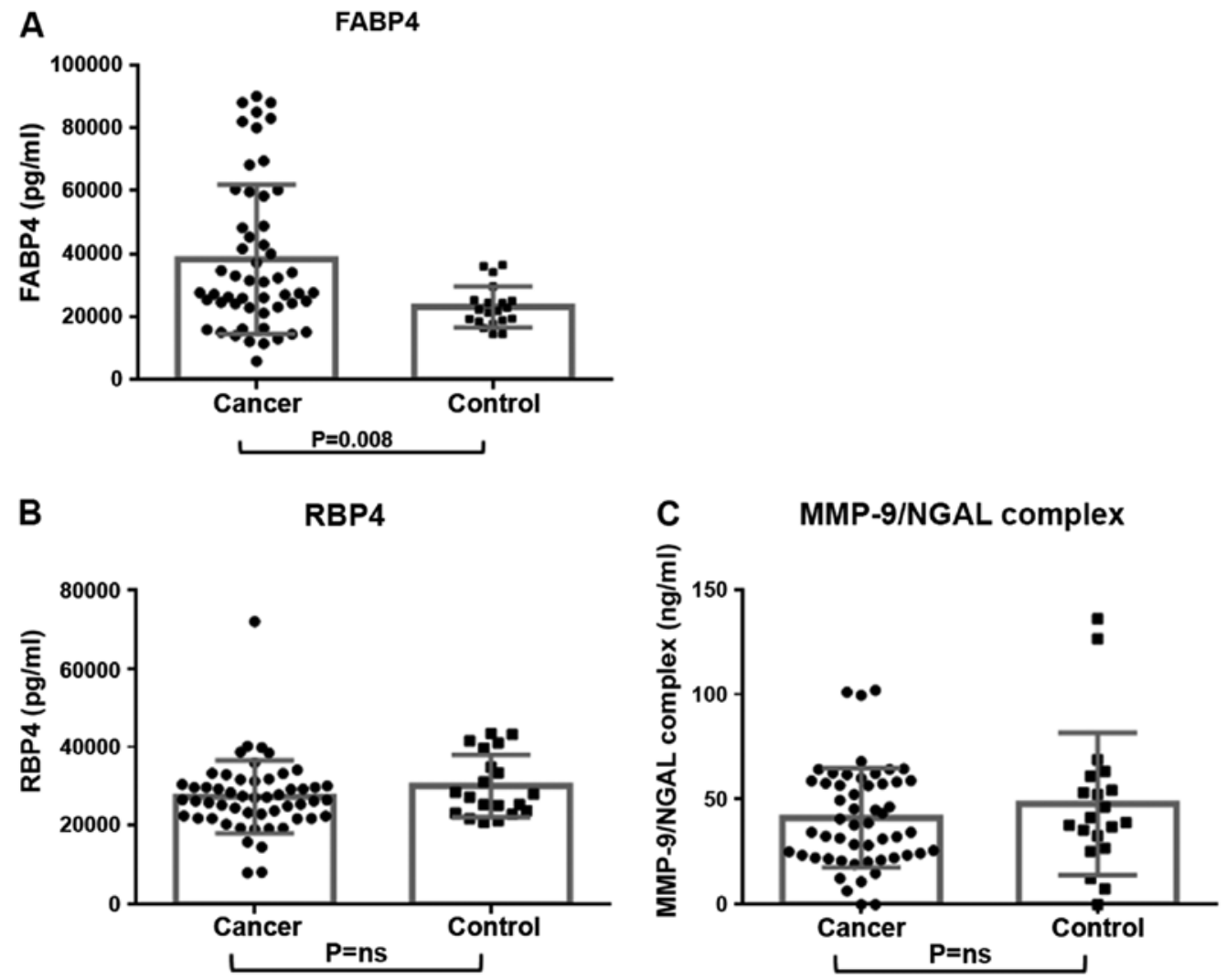

Figure 1. Association of breast cancer cases or control group patients and the expression of FABP4, RBP4 and MMP-9/NGAL. The concentration of the respective proteins are demonstrated, with bars indicating the mean with 95\% confidence interval. Serum concentrations of (A) FABP4, (B) RBP4 (C) MMP-9/NGAL were detected. FABP4, fatty acid-binding protein 4; RBP4, retinol binding protein 4; NGAL, neutrophil gelatinase-associated lipocalin.

A

FABP4

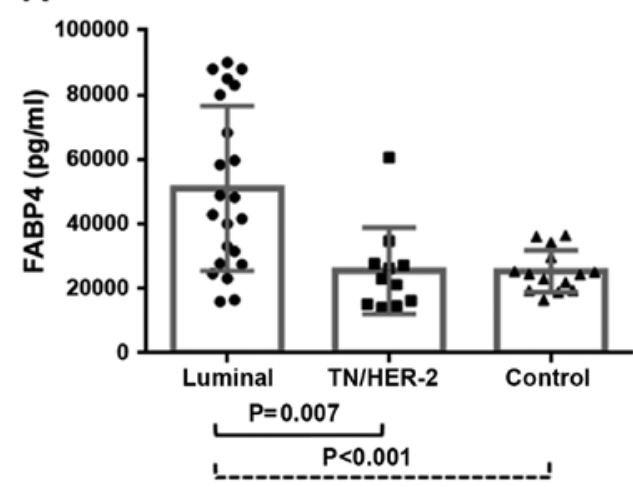

B

RBP4

C

MMP-9/NGAL complex
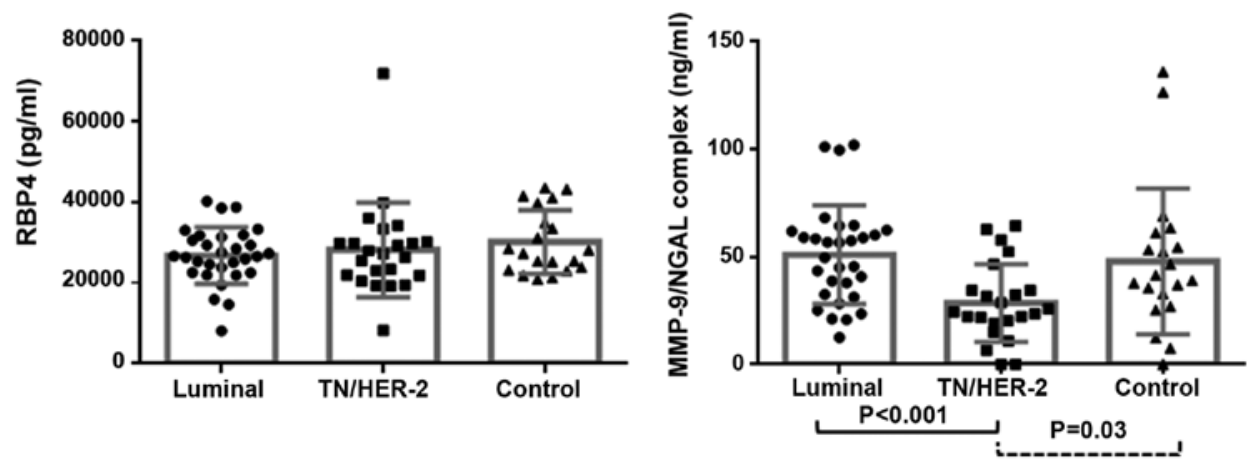

Figure 2. Association between breast cancer subtypes or control group patients and the expression of FABP4, RBP4 and MMP-9/NGAL. The concentration of the respective proteins are demonstrated, with bars indicating the mean with $95 \%$ confidence interval. Serum concentrations of (A) FABP4, (B) RBP4 and (C) the MMP-9/NGAL complex were detected. FABP4, fatty acid-binding protein 4; RBP4, retinol binding protein 4; NGAL, neutrophil gelatinase-associated lipocalin. 
Table IV. P-values were calculated to assess the differences in protein levels between patients and controls, and among the different breast cancer subtypes.

A, BMI $\geq 25 \mathrm{~kg} / \mathrm{m}^{2}$

\begin{tabular}{lrrc}
\hline & \multicolumn{3}{c}{ P-values } \\
\cline { 2 - 4 } Groups & FABP4 & RBP4 & $\begin{array}{c}\text { MMP-9/ } \\
\text { NGAL }\end{array}$ \\
\hline Breast cancer vs. control & 0.002 & 0.070 & 0.300 \\
Luminal vs. control & $<0.001$ & 0.100 & 0.800 \\
TN/HER-2 vs. control & 0.540 & 0.069 & 0.070 \\
Luminal vs. TN/HER-2 & 0.018 & 0.430 & 0.014 \\
\hline
\end{tabular}

B, BMI $<25 \mathrm{~kg} / \mathrm{m}^{2}$

the establishment of novel biomarkers that will enable the accurate prognosis and diagnosis of breast disease $(8,9,41,42)$. Hence, the present analysis focused on the study of serum FABP4, RBP4 and MMP9/NGAL complex levels in women diagnosed with breast cancer along with healthy women, while results were further associated with the different breast cancer molecular subtypes, BMI, menopause status diabetes and social background of patients.

In the present study differential serum levels of FABP4, RBP4 and MMP9/NGAL complex were detected in breast cancer cases, while different serum levels of the corresponding proteins were observed between the different molecular subtypes of breast disease, as well. In particular, increased serum concentration of FABP4 was detected in the examined breast cancer cases compared to control group, supporting previous findings $(10-12,17,43,44)$. Although substantially increased serum FABP4 levels were recorded in Luminal breast cancer samples, the concentration of the respective protein was found to be decreased in TN/HER-2 cases. These results imply that FABP4 may be a key element in the development of specific breast cancer molecular subtype and that each subtype uses different metabolic pathways. Indeed, receptor positive breast cancers are associated with gene signature involved in de novo lipogenesis, fat acid mobilization and oxidation while TNBC are associated with genes expressed in exogenous lipid uptake (45). This may support the hypothesis 

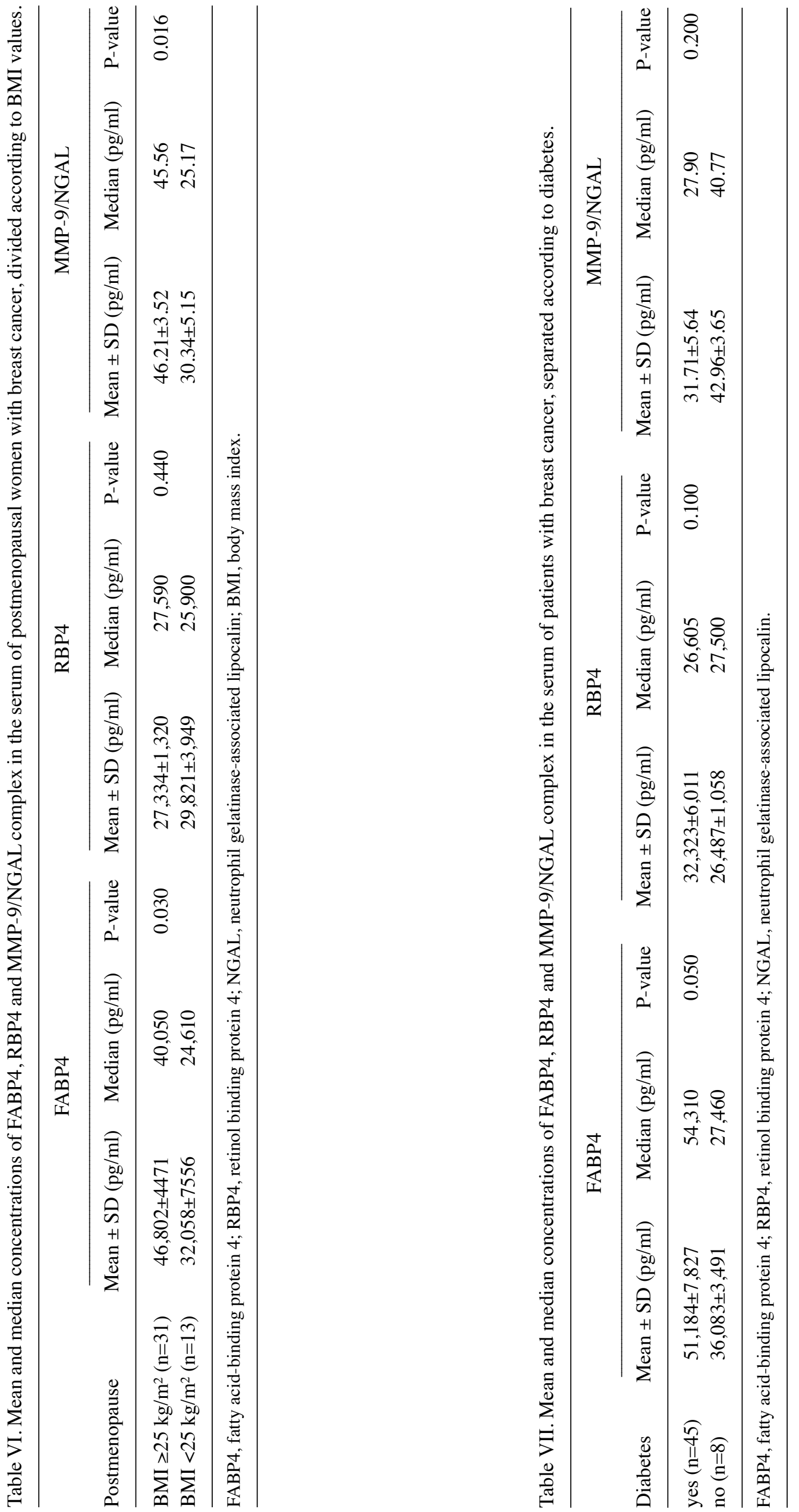
that specific metabolic lipid pathways are probably accelerated or moderated depending on tumor ER and HER-2 status. Interestingly, a previous study concerning the tissue analysis of breast tumors showed that the concentration of FABP4 was higher in HER-2 tumors and lower in Luminal A tumors, suggesting that this discrepancy lies mainly on differences in lipid metabolic pathways that have been observed in the different breast cancer subtypes $(43,46)$. Taking all these data into account it was anticipated that the different serum FABP4 levels that were detected in Luminal and TN/HER-2 breast cancers might be observed due to a possible stronger association between lipid metabolic status and breast cancer subtypes and therefore different levels of FABP4 might be circulating in the serum of patients. However, further analyses are required in order to confirm this hypothesis.

In regard to RBP4, previous research studies have recommended that RBP4 is not associated with breast cancer development, while the expression of RBP4 gene seems to be down regulated in breast cancer cases $(27,47)$. In contrast, a more recent study revealed for the first time that increased serum levels of RBP4 are related with breast cancer in menopausal women (28). In the present analysis no significant association between the serum levels of RBP4 and the development of breast cancer was observed in the tested patients $(27,47)$. Interestingly, a previous analysis by Formelli et al (27) suggested that low plasma RBP4 levels are found in postmenopausal women ( $\geq 55$ years old) and these patients exhibited poor prognosis of breast disease. In the present analysis, no significant association was recorded between serum levels of RBP4 and the development of breast cancer, while no significant relationship was described between RBP4 and menopausal status of the examined breast cancer patients.

Nowadays, little is known concerning the impact of MMP-9/NGAL complex on breast disease. A previous analysis derived by our group observed for the first time significantly increased serum levels of MMP-9/NGAL complex in breast cancer cases (29). In contrast to previous findings, we observed low levels of MMP-9/NGAL complex in breast cancer cases compared to healthy women but this association did not reach the limits of statistical significance. It is important to underline that significantly lower levels of MMP-9/NGAL complex were found in TN/HER-2 group compared to either healthy women or Luminal breast cancers $(\mathrm{P}<0.05)$. One possible explanation of the contradictory results could be the age group of the examined population, since in the previous study the mean age of the tested women was 52.8 years old, while in the present analysis the mean age of the examined patients was 62.8 years old (29). As a result, the age of the tested population may influence the MMP-9/NGAL complex levels $(48,49)$. An additional explanation could be the molecular subtype of breast cancer cases. Notably, in the previous analysis by our group the breast lesions were classified into four groups including sclerosis adenosis, atypical ductal hyperplasia, ductal carcinoma in situ and invasive carcinoma, while there was no available data concerning the molecular subtype of breast cancer (29). As a consequence the composition of breast cancer cases in TN/HER-2 subtype may influence the serum levels of MMP-9/NGAL complex and consequently might affect the prognostic value of MMP-9/NGAL complex in developing breast cancer. As a consequence, more analyses are needed in order to examine whether low serum MMP-9/NGAL complex levels might indicate the development of TN/HER-2 breast cancer in the examined women.

Finally, in order to assess the impact of obesity on the described associations, a stratified analysis based on BMI values and the serum levels of the corresponding proteins was carried out. According to our results it was revealed that FABP4 in patients with high BMI values (BMI $\geq 25 \mathrm{~kg} / \mathrm{m}^{2}$ ) is considerably increased, supporting previous findings (11). Moreover, patients diagnosed with Luminal breast cancer and BMI $\geq 25 \mathrm{~kg} / \mathrm{m}^{2}$ exhibited significantly higher levels of FABP4 $(\mathrm{P}<0.02)$. In addition, the serum levels of RBP4, MMP-9/NGAL complex are not influenced by BMI value, supporting previous studies $(28,29)$. However the concentration of MMP-9/NGAL complex was found to be significantly decreased in patients with TN/HER-2 breast cancer and BMI $\geq 25 \mathrm{~kg} / \mathrm{m}^{2}$ when compared to patients with Luminal subtype. It is significant to highlight that in patients with $\mathrm{BMI}<25 \mathrm{~kg} / \mathrm{m}^{2}$ the previously described associations did not reach the limits of statistical significance. Taking all these data into consideration it was suggested that BMI values regulate the described associations and thus BMI should be taken into consideration in order to draw more accurate conclusions concerning the prognosis of breast disease.

In addition, when protein serum levels were considered together with menopausal status, BMI and diabetes, it was observed that FABP4 concentration is significantly increased in postmenopausal patients with high BMI values as well as in breast cancer patients with diabetes, supporting previous findings $(11,17,50)$. Although FABP4 and estrogens have been associated with obesity and obesity-associated breast cancer development, a recent analysis proved that FABP4 and estrogens have an independent impact on obesity related breast cancer growth (51). Interestingly, MMP9/NGAL complex was significantly associated in breast cancer development in postmenopausal women. As it was described above no considerable association was found between MMP9/NGAL complex and breast cancer development. However, this association reaches the limits of statistical significance in postmenopausal women with BMI $\geq 25 \mathrm{~kg} / \mathrm{m}^{2}$. As a consequence, further analyses are required in order to confirm these outcomes as well as to examine whether MMP9/NGAL complex in association with estrogens contribute to the growth of breast cancer. To the best of our knowledge this is the first study that implicates MMP9/NGAL complex to the development of breast cancer in postmenopausal women.

Our results imply that there is a bidirectional interaction between circulating FABP4 and ER/PR receptors in tumor microenvironment. The relationship between lipid metabolism and breast cancer molecular subtypes varies considerably regarding menopausal status, BMI, presence of diabetes. Adipocytes and secreted fatted acid proteins induce resistance against to hormonal therapy, HER-2 targeting agents and radiotherapy (52). From a clinical perspective, the therapeutic interventions targeting multiple molecular pathways that are implicated in tumor establishment and progression could be more effective. That is to say the specific blockade of FABP4 may improve the efficacy of endocrine therapies and moderate resistance. Further studies should be conducted in order to better evaluate the complexity 
of lipid metabolism and the impact of obesity-related proteins as a diagnostic, prognostic and therapeutic tool in specific group of patients. This study has some limitations. The number of participants was small, while all specimens were collected from a single institute. As a consequence, further analyses are required in a larger population scale derived from different tertiary care Greek hospitals in order to reinforce our findings.

In conclusion, our results support the initial recommendation that FABP4 can be regarded as a powerful biomarker of breast cancer development, while its strong association with obesity indicates that FABP4 is a key therapeutic target for treatment of obesity-related breast cancer (17). To the best of our knowledge this is the first study that describes the association of serum FABP4 and MMP-9/NGAL levels in Luminal and TN/HER-2 breast cancer subtypes, respectively, suggesting that the measurement of these two factors may provide significant information concerning not only breast cancer development but also the growth of specific breast cancer subtype, in order to better evaluate the interaction of the respective circulating proteins in specific group of patients (postmenopausal, obese, diabetes). The present analysis anticipated that the metabolic profile of breast cancer subtype, the menopausal status and BMI of the examined women should be taken into consideration in order to select the most suitable biomarker, offering crucial information regarding the personalized prognosis, diagnosis and the proper therapy of breast disease.

\section{Acknowledgements}

Not applicable.

\section{Funding}

This work was financially supported by the Hellenic Anticancer Institute, a private non-profit organization. The funding source had no involvement in study design, conduct of research or manuscript publication.

\section{Availability of data and materials}

The datasets used and/or analyzed during the present study are available from the corresponding author on request.

\section{Author's contribution}

GB, EZ and FZ designed the present study. DB collected and analyzed samples, and interpreted results. GB, DT and EK analyzed and interpreted the data. DT and GB wrote the manuscript. All authors read and approved the final manuscript.

\section{Ethics approval and consent to participate}

All patients provided their written informed consent prior to enrollement. The current study was approved by the 'Saint Savvas' General Anticancer Hospital Ethics Committee (approval no. 1617017278).

\section{Patient consent for publication}

Not applicable.

\section{Competing interests}

The authors declare that they have no competing interests.

\section{References}

1. Bertucci F and Birnbaum D: Reasons for breast cancer heterogeneity. J Biol 7: 6, 2008.

2. Bray F, Ferlay J, Soerjomataram I, Siegel RL, Torre LA and Jemal A: Global cancer statistics 2018: GLOBOCAN estimates of incidence and mortality worldwide for 36 cancers in 185 countries. CA Cancer J Clin 68: 394-424, 2018.

3. Bray F, Ferlay J, Laversanne M, Brewster DH, Gombe Mbalawa C, Kohler B, Piñeros M, Steliarova-Foucher E, Swaminathan R, Antoni S, et al: Cancer incidence in five continents: Inclusion criteria, highlights from Volume $\mathrm{X}$ and the global status of cancer registration. Int J Cancer 137: 2060-2071, 2015.

4. Harbeck N, Penault-Llorca F, Cortes J, Gnant M, Houssami N, Poortmans P, Ruddy K, Tsang J and Cardoso F: Breast cancer. Nat Rev Dis Primers 5: 66, 2019.

5. DeSantis C, Ma J, Bryan L and Jemal A: Breast cancer statistics, 2013. CA Cancer J Clin 64: 52-62, 2014.

6. Early Breast Cancer Trialists' Collaborative Group (EBCTCG): Effects of chemotherapy and hormonal therapy for early breast cancer on recurrence and 15-year survival: An overview of the randomised trials. Lancet 365: 1687-1717, 2005.

7. Colomer R, Aranda-Lopez I, Albanell J, García-Caballero T, Ciruelos E, López-García MÂ, Cortés J, Rojo F, Martín M and Palacios-Calvo J: Biomarkers in breast cancer: A consensus statement by the Spanish Society of Medical Oncology and the Spanish Society of Pathology. Clin Transl Oncol 20: 815-826, 2018.

8. Kashyap D and Kaur H: Cell-free miRNAs as non-invasive biomarkers in breast cancer: Significance in early diagnosis and metastasis prediction. Life Sci 246: 117417, 2020.

9. Bourgain C, Pourtau L, Mazouni C, Bungener M and Bonastre EJ: Imperfect biomarkers for adjuvant chemotherapy in early stage breast cancer with good prognosis. Soc Sci Med 246: 112735, 2020.

10. Guaita-Esteruelas S, Bosquet A, Saavedra P, Gumà J, Girona J, Lam EW, Amillano K, Borràs J and Masana L: Exogenous FABP4 increases breast cancer cell proliferation and activates the expression of fatty acid transport proteins. Mol Carcinog 56: 208-217, 2017.

11. Guaita-Esteruelas $S$, Saavedra-Garcia $P$, Bosquet $A$, Borràs $J$, Girona J, Amiliano K, Rodríguez-Balada M, Heras M, Masana L and Gumà J: Adipose-derived fatty acid-binding proteins plasma concentrations are increased in breast cancer patients. Oncologist 22: 1309-1315, 2017.

12. Guaita-Esteruelas S, Guma J, Masana L and Borras J: The peritumoural adipose tissue microenvironment and cancer. The roles of fatty acid binding protein 4 and fatty acid binding protein 5 . Mol Cell Endocrinol 462: 107-118, 2018.

13. Zimmerman AW and Veerkamp JH: New insights into the structure and function of fatty acid-binding proteins. Cell Mol Life Sci 59: 1096-1116, 2002.

14. Zeng J, Sauter ER and Li B: FABP4: A new player in obesity-associated breast cancer. Trends Mol Med 26: 437-440, 2020.

15. Prentice KJ, Saksi J and Hotamisligil GS: Adipokine FABP4 integrates energy stores and counterregulatory metabolic responses. J Lipid Res 60: 734-740, 2019.

16. Cao H, Sekiya M, Ertunc ME, Burak MF, Mayers JR, White A, Inouye K, Rickey LM, Ercal BC, Furuhashi M, et al: Adipocyte lipid chaperone AP2 is a secreted adipokine regulating hepatic glucose production. Cell Metab 17: 768-778, 2013.

17. Hao J, Zhang Y, Yan X, Yan F, Sun Y, Zeng J, Waigel S, Yin Y, Fraig MM, Egilmez NK, et al: Circulating adipose fatty acid binding protein is a new link underlying obesity-associated breast/mammary tumor development. Cell Metab 28: 689-705 e5, 2018.

18. Ginestier C, Hur MH, Charafe-Jauffret E, Monville F, Dutcher J, Brown M, Jacquemier J, Viens P, Kleer CG, Liu S, et al: ALDH1 is a marker of normal and malignant human mammary stem cells and a predictor of poor clinical outcome. Cell Stem Cell 1: 555-567, 2007.

19. Peek ME, Bhatnagar A, McCarty NA and Zughaier SM: Pyoverdine, the major siderophore in pseudomonas aeruginosa, evades NGAL recognition. Interdiscip Perspect Infect Dis 2012: 843509, 2012.

20. Flowe DR: The lipocalin protein family: Structure and function. Biochem J 318: 1-14, 1996. 
21. Kawaguchi R, Yu J, Honda J, Hu J, Whitelegge J, Ping P, Wiita P, Bok D and Sun H: A membrane receptor for retinol binding protein mediates cellular uptake of vitamin A. Science 315: 820-825, 2007.

22. Berry DC, Jin H, Majumdar A and Noy N: Signaling by vitamin $\mathrm{A}$ and retinol-binding protein regulates gene expression to inhibit insulin responses. Proc Natl Acad Sci USA 108: 4340-4345, 2011.

23. Norseen J, Hosooka T, Hammarstedt A, Yore MM, Kant S, Aryal P, Kiernan UA, Phillips DA, Maruyama H, Kraus BJ, et al: Retinol-binding protein 4 inhibits insulin signaling in adipocytes by inducing proinflammatory cytokines in macrophages through a c-Jun N-terminal kinase- and toll-like receptor 4-dependent and retinol-independent mechanism. Mol Cell Biol 32: 2010-2019, 2012.

24. Abola MV, Thompson CL, Chen Z, Chak A, Berger NA, Kirwan JP and Li L: Serum levels of retinol-binding protein 4 and risk of colon adenoma. Endocr Relat Cancer 22: L1-L4, 2015.

25. Cheng Y, Liu C, Zhang N, Wang S and Zhang Z: Proteomics analysis for finding serum markers of ovarian cancer. Biomed Res Int 2014: 179040, 2014

26. Chen Y, Azman SN, Kerishnan JP, Zain RB, Chen YN, Wong YL and Gopinath SC: Identification of host-immune response protein candidates in the sera of human oral squamous cell carcinoma patients. PLoS One 9: e109012, 2014.

27. Formelli F, Meneghini E, Cavadini E, Camerini T, Di Mauro MG De Palo G, Veronesi U, Berrino F and Micheli A: Plasma retinol and prognosis of postmenopausal breast cancer patients. Cancer Epidemiol Biomarkers Prev 18: 42-48, 2009.

28. Jiao C, Cui L, Ma A, Li N and Si H: Elevated Serum Levels of Retinol-Binding Protein 4 Are Associated with Breast Cancer Risk: A Case-Control Study. PLoS One 11: e0167498, 2016.

29. Provatopoulou X, Gounaris A, Kalogera E, Zagouri F, Flessas I, Goussetis E, Nonni A, Papassotiriou I and Zografos G: Circulating levels of matrix metalloproteinase-9 (MMP-9), neutrophil gelatinase-associated lipocalin (NGAL) and their complex MMP-9/NGAL in breast cancer disease. BMC Cancer 9: 390, 2009.

30. Barasch J, Hollmen M, Deng R, Hod EA, Rupert PB, Abergel RJ, Allred BE, Xu K, Darrah SF, Tekabe Y, et al: Disposal of iron by a mutant form of lipocalin 2. Nat Commun 7: 12973, 2016.

31. Chakraborty S, Kaur S, Guha S and Batra SK: The multifaceted roles of neutrophil gelatinase associated lipocalin (NGAL) in inflammation and cancer. Biochim Biophys Acta 1826: 129-169, 2012.

32. Candido S, Abrams SL, Steelman LS, Lertpiriyapong K, Fitzgerald TL, Martelli AM, Cocco L, Montalto G, Cervello M, Polesel J, et al: Roles of NGAL and MMP-9 in the tumor microenvironment and sensitivity to targeted therapy. Biochim Biophys Acta 1863: 438-448, 2016.

33. Torti SV and Torti FM: Iron and cancer: More ore to be mined. Nat Rev Cancer 13: 342-355, 2013.

34. Roli L, Pecoraro V and Trenti T: Can NGAL be employed as prognostic and diagnostic biomarker in human cancers? A systematic review of current evidence. Int J Biol Markers 32: e53-e61, 2017.

35. Chen YC, Chang SC, Huang YH, Lee YJ, Chang CC, Liao JW and Hsu WL: Expression and the molecular forms of neutrophil gelatinase-associated lipocalin and matrix metalloproteinase 9 in canine mammary tumours. Vet Comp Oncol 17: 427-438, 2019.

36. Yan L, Borregaard N, Kjeldsen L and Moses MA: The high molecular weight urinary matrix metalloproteinase (MMP) activity is a complex of gelatinase B/MMP-9 and neutrophil gelatinase-associated lipocalin (NGAL). Modulation of MMP-9 activity by NGAL. J Biol Chem 276: 37258-37265, 2001.
37. Sung H, Choi JY, Lee SA, Lee KM, Han S, Jeon S, Song M, Lee Y, Park SK, Yoo KY, et al: The association between the preoperative serum levels of lipocalin-2 and matrix metalloproteinase-9 (MMP-9) and prognosis of breast cancer. BMC Cancer 12: 193, 2012.

38. Huang H: Matrix Metalloproteinase-9 (MMP-9) as a cancer biomarker and MMP-9 biosensors: Recent advances. Sensors (Basel) 27: 3249, 2018.

39. Goldhirsch A, Winer EP, Coates AS, Gelber RD, Piccart-Gebhart M, Thürlimann B and Senn HJ; Panel members: Personalizing the treatment of women with early breast cancer: Highlights of the St gallen international expert consensus on the primary therapy of early breast cancer 2013. Ann Oncol 24 : 2206-2223, 2013.

40. Goldhirsch A, Wood WC, Coates AS, Gelber RD, Thürlimann B and Senn HJ; Panel members: Strategies for subtypes-dealing with the diversity of breast cancer: Highlights of the St. Gallen international expert consensus on the primary therapy of early breast cancer 2011. Ann Oncol 22: 1736-1747, 2011.

41. Waks AG and Winer EP: Breast cancer treatment: A Review. JAMA 321: 288-300, 2019.

42. Fragomeni SM, Sciallis A and Jeruss JS: Molecular Subtypes and Local-Regional Control of Breast Cancer. Surg Oncol Clin N Am 27: 95-120, 2018

43. Kim S, Lee Y and Koo JS: Differential expression of lipid metabolism-related proteins in different breast cancer subtypes. PLoS One 10: $\mathrm{e} 0119473,2015$.

44. Wu Q, Li B, Li Z, Li J, Sun S and Sun S: Cancer-associated adipocytes: key players in breast cancer progression. J Hematol Oncol 12: 95, 2019.

45. Koundouros $\mathrm{N}$ and Poulogiannis G: Reprogramming of fatty acid metabolism in cancer. Br J Cancer 122: 4-22, 2020.

46. Moreno-Sanchez R, Rodriguez-Enriquez S, Marin-Hernandez A and Saavedra E: Energy metabolism in tumor cells. FEBS J 274: 1393-1418, 2007

47. Merdad A, Karim S, Schulten HJ, Jayapal M, Dallol A, Buhmeida A, Al-Thubaity F, GariI MA, Chaudhary AG, Abuzenadah AM and Al-Qahtani MH: Transcriptomics profiling study of breast cancer from Kingdom of Saudi Arabia revealed altered expression of Adiponectin and Fatty Acid Binding Protein4: Is lipid metabolism associated with breast cancer? BMC Genomics 16 (Suppl 1): S11, 2015.

48. Lanning NJ, Castle JP, Singh SJ, Leon AN, Tovar EA, Sanghera A, MacKeigan JP, Filipp FV and Graveel CR: Metabolic profiling of triple-negative breast cancer cells reveals metabolic vulnerabilities. Cancer Metab 5: 6, 2017.

49. Tayyari F, Gowda GAN, Olopade OF, Berg R, Yang HH, Lee MP, Ngwa WF, Mittal SK, Raftery D and Mohammed SI: Metabolic profiles of triple-negative and luminal A breast cancer subtypes in African-American identify key metabolic differences. Oncotarget 9: 11677-11690, 2018.

50. Hancke K, Grubeck D, Hauser N, Kreienberg R and Weiss JM: Adipocyte fatty acid-binding protein as a novel prognostic factor in obese breast cancer patients. Breast Cancer Res Treat 119: 367-367, 2010.

51. Li B, Hao J, Yan X, Kong M and Sauter ER: A-FABP and oestrogens are independently involved in the development of breast cancer. Adipocyte 8: 379-385, 2019.

52. Choi J, Cha YJ, Koo JS: Adipocyte biology in breast cancer: From silent bystander to active facilitator. Prog Lipid Res 69: 11-20, 2018.

This work is licensed under a Creative Commons Attribution-NonCommercial-NoDerivatives 4.0 International (CC BY-NC-ND 4.0) License. 